\title{
THANK GOD FOR LAB: SYSTEMIC LUPUS ERYTHEMATOSUS - A DIFFICUIT DIAGNOSIS, A DIFFICUIT DISEASE
}

\section{Tica Irina', Nicoară Alina-Doina', Șuța Gristina', Ghinea Mihaela-Maria', Tica Vlad-Iustin', Niculescu Zizi'}

${ }^{1}$ Faculty of Medicine, University "Ovidius" of Constanța

Systemic Lupus Erythematosus (SLE) involves autoimmune mechanisms that affect multiple systems and has variable clinical manifestations. It affects mainly feminine gender, usually of child-bearing age. Very late onset SLE (patients older than 75 years) is rarely met and difficult to diagnose due to the unspecific symptoms. The diagnosis is frequently mistaken for drug-induced SLE or for other age-specific disorders.

We present the case of a 78 years old male patient complaining of hemoptysis in small amounts, fever, dyspnea at small efforts, weight loss, and important asthenia. Based on physical examination, severe anemia and thoracic CT aspect, several diagnosis were taken into account: anemic syndrome and pleurisy due to refractory pneumonia, pulmonary neoplasm, pulmonary tuberculosis, congestive heart failure and Wegener granulomatosis. Only when direct Coombs test came positive and all the other serological tests resulted negative, diagnosis of SLE was considered. Confirmation came along with high titers of antinuclear antibodies, anti double strained DNA antibodies and lupic anticoagulant and low levels of C3c and C4.

We conclude that the diagnosis of SLE must be taken into consideration even in elder male patients. Therapeutic options in these patients are challenging because of the associated comorbidities and side effects.

Keywords: SLE, hemoptysis, pleurisy, anemia, hemolysis, anti-ds DNA antibodies

\section{Introduction}

Systemic Lupus Erythematosus (SLE) involves autoimmune mechanisms that affect multiple systems and has variable clinical manifestations and has variable clinical manifestations.

SLE usually affects feminine gender of child-bearing age, mostly African-Americans and less Caucasians (1).

When patients' age at onset is over 50-65 years old (less than $20 \%$ of the cases with SLE), it is considered late-onset lupus. Only a few cases with onset ages higher than 75 years old are reported in medical literature (2-4).
The clinical aspect of late-onset SLE is different from the one of the young patients, often misdiagnosed as drug-induced SLE, with nonspecific symptoms such as anorexia, weight loss, fatigue, arthralgias, myalgias, pyrexia and cognitive dysfunction (5-8). As a result, the diagnosis is difficult and delayed in this group of patients.

Explanations for the peculiar clinical and laboratory manifestations of late-onset LES are based on sex hormone implication (female: male sex ratio declines from a 10:1 ratio in the younger patient to a 4:1 ratio in the older patient) (9), race (especially Caucasian and black), age-related immunological modifications: the production 
of $\mathrm{B}$ cells diminishes while the production of autoantibodies increases (rheumatoid factor, antibodies anti-Ro, anti-La), along with proinflammatory cytokines (tumor necrosis factor, IL-6) $(10,11)$.

Treatment in these patients is challenging as comorbidities are usually associated, while SLE specific treatment may increase the complications of the associated diseases or the side effects of other medication.

\section{Gase report}

We present the case of a 78 years old patient, male, who came to emergency room of the District Clinical Emergency Hospital of Constanta, in August 2017 for daily hemoptysis in small amounts, fever, dyspnea at small efforts, weight loss, and important asthenia.

We found out from his history that he had these symptoms for about 2 months and that he was hospitalized 1 month ago and diagnosed with left lobar pneumonia and discharged after 2 weeks of antibiotic treatment and improved clinical aspect. He was suffering, also, from Diabetes mellitus type 2, essential hypertension, arthralgias in knee joints, nodular subclinical hypothyroid goiter. His wife recognized patient's cognitive decline in the last 2 months. In the last month, he performed an esogastroduodenoscopy because of dysphagia, but with no pathological results.

Mention should be made that an informed consent had been previously obtained from the patient and the presentation was approved by the Institutional Ethical Committee.

Physical examination at admition revealed a pale patient, underweight, with discreet edema of the lower limbs, with crepitant rales over the left superior lobe and abolished breathing sounds in both pulmonary base, tachycardic (90 beats/ min), normal blood pressure $(100 / 70 \mathrm{mmHg})$, spontaneously bleeding gums, especially in the morning, in rest normal (Figure 1).

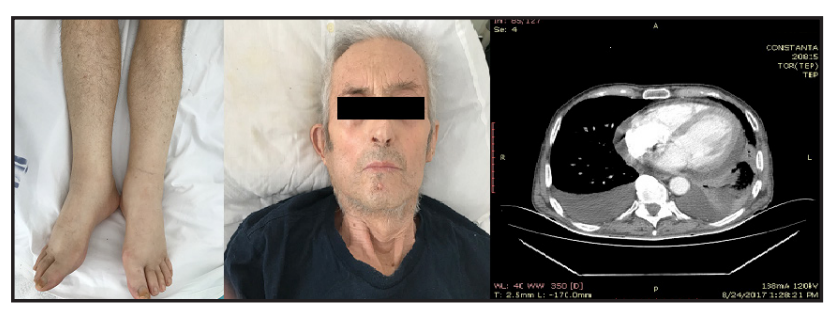

Figure 1. Clinical aspect and thoracic CT revealing pleurisy, pericarditis and consolidation pulmonary areas.

Laboratory evaluation at admition revealed moderate normochromic, normocytic anemia, discreet lymphopenia, mild hyposideremia, very important inflammatory syndrome (elevated erythrocyte sedimentation rate - ESR and C reactive protein - CRP). Biological parameters at admition are revealed in Tables 1 and 2.

Chest X-ray and thoracic computed tomography (CT) performed at admition revealed alveolar consolidation areas in the inferior and superior left lobes, bilateral basal pleurisy, pericarditis, nodular goiter (Figure 1).

Based on clinical, biological and radiological aspects, the diagnosis of pneumonia was reiterated and antibiotic treatment (cephalosporin and ciprofloxacin for seven dayswhile waiting for sputum cultures results) along with haemostatic and diuretic treatment was initiated.

Sputum results came irrelevant (negative direct examination for Koch bacillus, no pathogenic flora, no atypical cells), cultures negative for pathogenic flora, pleural fluid examination proved to be transudate, with insignificant increased level of adenosine deaminase (ADA).

In the mean time, due to the history of hypertension, the presence of edema, of dyspnea at small efforts and of pericarditis, cardiologic examination and cardiac ultrasound were performed. The diagnosis of heart failure II $^{\text {nd }}$ NYHA class with reduced left ventricle output (40\%), essential hypertension, mitral and aortic insufficiency, important pulmonary hypertension and minimal pericarditis was established. Specific treatment with: association of loop diuretics and spironolactone, selective beta-blockers, Angiotensin II receptor antagonists (ARBs), and paracetamol was recommended.

Despite the treatment, patient's situation was slowly deteriorating: hemoptysis continued, 
Table 1. Biological parameters

\begin{tabular}{|c|c|c|c|c|c|c|c|}
\hline Parameters/days from admition & Day 1 & Day 2 & Day 4 & Day 5 & Day 12 & Day 19 & Day28 \\
\hline Hemoglobin (nv: 3.8-5.8 g/dL) & 6.9 & 7.1 & 6.1 & & & 8 & 9 \\
\hline VEM (nv: 81-103 fL) & 86 & 86.7 & 88.2 & & & & 89.9 \\
\hline White blood count (nv: 4-10x1000/mL) & 2.13 & 5.11 & 4.02 & & & 5.21 & 8.08 \\
\hline Lymphocytes (nv: $1-4$ x1000/mL) & 1.55 & 0.7 & 0.44 & & & 0.77 & 1.49 \\
\hline Platelets (nv: $150-450 \times 1000 / \mathrm{mL})$ & 199 & 311 & 291 & & & 267 & 287 \\
\hline Sideremia (nv: $59-158 \mu \mathrm{g} / \mathrm{dL}$ ) & 24 & & & & & & \\
\hline ESR (nv: $<20 \mathrm{~mm} / \mathrm{h}$ ) & 77 & & & & 91 & 49 & \\
\hline CRP (nv: $<0.5 \mathrm{mg} / \mathrm{dL})$ & 4.41 & & & & & & \\
\hline Creatinine (nv: $<1.2 \mathrm{mg} / \mathrm{dL})$ & 1.03 & & & 0.97 & & 0.83 & \\
\hline ASAT (nv: $<40 \mathrm{U} / \mathrm{L})$ & 24 & & & & & 19 & \\
\hline ALAT (nv: <41 U/L) & 12 & & & & & 14 & \\
\hline LDH (nv: 135-225 U/L) & & 245 & & & & & \\
\hline Total serum proteins (nv: $6.6-8.7 \mathrm{~g} / \mathrm{dL}$ ) & & & 6.1 & & & & \\
\hline Serum albumine (nv: $3.5-5.2 \mathrm{~g} / \mathrm{dL})$ & & & 2.6 & & & & \\
\hline Urinary proteins/24 H (nv: $<140 \mathrm{mg} / 24 \mathrm{~h})$ & & & 241.8 & & & & \\
\hline TSH (nv: $0.4-4.2 \mu U I / m L)$ & 5.1 & & & & & & \\
\hline FT4 free thyroxine (nv: $10.6-22.7 \mathrm{pmol} / \mathrm{L}$ ) & 19.3 & & & & & & \\
\hline Pleural fluid examination & \multicolumn{7}{|c|}{$\begin{array}{l}\text { Amylase: } 32 \mathrm{UI} / \mathrm{L} \text {; LDH: } 112 \mathrm{mg} / \mathrm{dL} \text {, Proteins: } 2.6 \mathrm{~g} / \mathrm{dL}, \mathrm{ADA}<45 \\
\mathrm{UI} / \mathrm{L} \text {, presence of inflammatory cells(lymphocytes, monocytes, } \\
\text { granulocytes) and epithelial cells with possible inflammatory cyto- } \\
\text { nuclear modifications }\end{array}$} \\
\hline Sputum examination & \multicolumn{7}{|c|}{$\begin{array}{l}\text { Negative Koch bacillus, frequent squamous epithelial cells, frequent } \\
\text { leukocytes, frequent fungi, frequent saprophytic bacterial flora }\end{array}$} \\
\hline Bronchial aspiration fluid & \multicolumn{7}{|c|}{$\begin{array}{l}\text { Negative Koch bacillus, no pathogenic flora, exclusive typical } \\
\text { squamous epithelial cells }\end{array}$} \\
\hline
\end{tabular}

Table 2. Immunologic and tumor markers

\begin{tabular}{|c|c|c|c|c|c|c|}
\hline Parameters/days from admition & Day 1 & Day 2 & Day 4 & Day 5 & Day 12 & Day 19 \\
\hline Cyfra 21-1 (nv: <3.3 ng/mL) & 5.6 & & & & & \\
\hline NSE (nv: $<17$ ng/mL) & 7.94 & & & & & \\
\hline ATPO (nv: <34 UI/mL) & 17 & & & & & \\
\hline ATGB (nv: $<115 \mathrm{UI} / \mathrm{mL})$ & 14 & & & & & \\
\hline Calcitonin(nv: $<14.3 \mathrm{pg} / \mathrm{mL})$ & 4 & & & & & \\
\hline Gamma globulins (nv: 0.6-1.6 g/dL) & & & 1.7 & & & \\
\hline cANCA $(\mathrm{nv}<7 \mathrm{U} / \mathrm{ml})$ & & & & 0.4 & & \\
\hline $\begin{array}{l}\text { IgG anti Mycoplasma pneumoniae (nv: }<9 \\
\text { AU/mL) }\end{array}$ & & & & $<9$ & & \\
\hline $\begin{array}{l}\text { gM anti Mycoplasma pneumoniae } \\
(\mathrm{nv}:<09 \text { Index) }\end{array}$ & & & & $<0.9$ & & \\
\hline $\begin{array}{l}\text { IgA anti Mycoplasma pneumoniae (nv: }<9 \\
\text { AU } / \mathrm{mL} \text { ) }\end{array}$ & & & & $<9$ & & \\
\hline anti dsDNA (nv: $<100 \mathrm{ui} / \mathrm{mL})$ & & & & & 2990.8 & \\
\hline ANA (nv: titer<1/80) & & & & & $1 / 2560$ & \\
\hline Lupic anticoagulant (nv: negative) & & & & & & positive \\
\hline C3c (nv: $90-180 \mathrm{mg} / \mathrm{dL})$ & & & & & & 64 \\
\hline C4 (nv: $10-40 \mathrm{mg} / \mathrm{dL})$ & & & & & & 8 \\
\hline $\begin{array}{l}\text { Anticardiolipinic antibodies IgG } \\
\text { (nv: }<20 \mathrm{GPL} / \mathrm{mL} \text { ) }\end{array}$ & & & & & & 6 \\
\hline Immunohaematology & \multicolumn{6}{|c|}{$\begin{array}{l}\text { ABO Group: A, Rh(D): positive; irregular antibodies: positive, direct } \\
\text { Coombs test: positive, Erythrocyte phenotype: RH/KEL: CcDWee, Kell } \\
\text { negative, TCD: positive }(2+)\end{array}$} \\
\hline
\end{tabular}


edema increased, anemia aggravated, asthenia worsened and nocturnal disorientation episodes occurred.

Occult blood testing from stools was performed with negative result (patient refused colonoscopy).

Because of persistent hemoptysis we performed bronchoscopy which confirmed the presence of small blood clots in the left principal bronchi but did not find any suspect lesion. Aspirate of the bronchial lavage was sampled but no relevant laboratory results came from it.

Under these circumstances, in need for blood transfusion, direct Coombs test and serum lactic dehydrogenase were performed with positive results (see Table no 2).

One unit of typed and cross-matched packed red blood cells transfusion was performed, with a slight improvement in patient general status and in the level of hemoglobin (from $6.8 \mathrm{~g} / \mathrm{dL}$ to $7 \mathrm{~g}$ / dL).

Control inflammatory tests maintained at high levels, nephritic proteinuria was detected with subsequent hipoalbuminemia, but normal renal function (see Table no 1).

We continued antibiotic treatment, under the pneumologist recommendation, changing the large spectrum antibiotics (now: meropenem and moxifloxacine) for about 10 days, in association with dexamethasone $8 \mathrm{mg}$ per day, with no improvement in patient's condition.

Other etiologies for hemoptysis, like Wegener granulomatosis and pneumonia with Mycoplasma pneumoniae, were considered. Immunoglobulines $\mathrm{A}, \mathrm{M}$ and $\mathrm{G}$ against Mycoplasma pneumoniae and anti-neutrophil cytoplasmic antibodies (c ANCA) came within normal limits.

Finally, based on the presence of hemolytic anemia, serositis, positive inflammatory tests, SLE was taken into discussion. Antinuclear antibodies (ANA) and anti double strained DNA antibodies (anti-dsDNA) were performed and results came positive in very high titers (see Table 1).

Rheumatologic examination requested other SLE specific tests: lupic anticoagulant with positive result, levels of $\mathrm{C} 3 \mathrm{c}$ and $\mathrm{C} 4$ - lower levels than normal, immunoglobulin $G$ anti cardiolipin - negative result, anti-Sm antibodies
- negative results.

In the end, the patient's diagnosis was: Systemic Lupus Erythematosus - SLE with hematologic, pulmonary and, possible, neurologic manifestations, based on Systemic Lupus International Collaborating Clinics group (SLICC) (2012) and American College of Rheumatology (ACR) criteria of diagnosis for SLE.

Pulse therapy with $3 \mathrm{~g}$ of Solu-Medrol was performed followed by treatment with hydroxychloroquine $200 \mathrm{mg}$ twice per day (under the control of fundus oculi examinations) and medrol $1 \mathrm{mg} / \mathrm{kg} /$ day, along with gastroprotective treatment, antihypertensive drugs, oral anti diabetic treatment, vitamin D 2000 IU per day, calcium $500 \mathrm{mg}$ per day and oral lavage with chlorhexidine.

After 7 days on the above mentioned treatment, hemoptysis stopped, edema diminished, asthenia improved, hemoglobin levels raised up to $9 \mathrm{~g} / \mathrm{dL}$. Thoracic control CT was performed and the alveolar consolidation processes were reduced in area as well as the pleural and pericardic fluid; the patient was discharged with indication of continuing hydroxychloroquine $200 \mathrm{mg}$ twice per day and to tapper the dosage of medrol with $8 \mathrm{mg}$ every 2 weeks.

\section{Discussions}

We did not expect the diagnosis of SLE in a 78 years old male patient presented for hemoptysis, weight loss and asthenia.

SLE affects mainly women, at child-bearing age. The prevalence of SLE varies: in Turkey $51.7 / 100,000$ inhabitants (12), in USA between 6 up to 241 cases per 100,000 inhabitants, with an important increase of incidence in the last 4 decades due to improvements in diagnosing the mild forms of disease (13). The incidence of lateonset SLE varies between $12 \%$ and $18 \%$, but patients included in these studies are considered with late-onset if they are older than 50-65 years old, not older than 75 years old (14). From our knowledge, just a few cases of patients with onset of SLE at ages older than 75 years are published, and none with hemoptysis as onset at this age (24). 
Clinical presentation of this specific group of patients is different from the young onset ones. The disease onset is insidious, rarely with skin manifestations as malar rash and discoid lupus or with renal impairment. They present more frequently fever, serositis, sicca syndrome, arthralgia, pulmonary disorder and neuropsychiatric symptoms.

However, despite of population aging and all the new information about SLE, there is still little information about the evolution and treatment of this disease in old patients.

The diagnosis is frequently mistaken for drug- induced SLE in patients receiving multiple treatments for other diseases, and delayed due to the nonspecific symptoms and signs. Many of the clinical manifestations can be considered due to other specific disorders met in elderly: heart failure, bronchopulmonary neoplasm, chronic kidney diseases, etc.

The unspecific clinical presentation was the case of our patient also, he complained of asthenia, anorexia, weight loss and hemoptysis. Based on these symptoms and signs at this age, we took primarily into consideration the diagnosis of pulmonary neoplasm, which was not confirmed either by the thoracic CT, bronchoscopy, examinations of pleural fluid and of the fluid from pulmonary lavage or pulmonary neoplasm markers (NSE and Cyfra 21-1).

Second, we took into consideration the diagnosis of pulmonary tuberculosis, again not sustained by sputum examinations (cultures, cytology), ADA in pleural fluid, pulmonary CT aspect.

We considered that heart failure can be responsible for hemoptysis but this was not consistent with its presence each morning for 2 months and with the significant weight loss and loss of appetite.

Oral lavage with chlorhexidine reduced to minimum the gingivorragias which might have been mistaken for hemoptysis in our diabetic patient with deteriorated neurological status. We noticed improving in periodontal lesions, similar to literature data (15).

Other rare causes of hemoptysis were taken into consideration as: Wegener granulomatosis (unsustained by normal serum cANCA level) and pneumonia with Mycoplasma Pneumoniae (negative serological examinations).

The moment the patient status required blood transfusion, we discovered hemolytic anemia. That was the moment when, taken serositis into account also, we finally thought about SLE. The diagnosis of SLE was established on 4 SLICC and ACR criteria (serositis, hemolytic anemia, lymphopenia, presence of ANA and anti-dsDNA antibodies, low C3 and C4c levels, possible neurologic impairment) and specific treatment was initiated.

Pulmonary manifestations in SLE include pulmonary hypertension (found by cardiac ultrasound performed in our patient), lupus pneumonitis (revealed by thoracic CT). Other pulmonary manifestations can be: chronic lupus interstitial lung disease, complementmediated pulmonary leukoaggregation, alveolar hemorrhage, or infection (16). Our patient did not have the typical aspect of lupus pleurisy: exudative, with an elevated pleural lactate dehydrogenase level, but a transudate with normal LDH level. We were afraid that diffuse alveolar hemorrhage may follow hemoptysis, but, fortunately, it did not, as, even rare, it is considered a life-threatening pulmonary complication of SLE

We did not find in medical literature patients with late onset SLE having hemoptysis as main manifestation.

Arthralgias of the knees in our patient were considered on arthrosis basis and not lupus related. A future reference for balneal therapy will be taken into consideration once the acute flare stops. It is of interest to find if a correlation exists between the levels of serum adiponectin in this patient, previously and after balneal treatment, as we have the knowledge that adiponectine's levels in SLE are higher than normal (17) and that balneal therapy with cold mud applications also elevates adiponectine's level (18).

Another peculiarity of our case is that although it has been reported that anti-dsDNA and hypocomplementaemia are present less frequently in late onset-SLE, our patient presented anti-dsDNA antibodies at very high levels, and very low levels of C3c and C4 $(19,20)$.

The treatment in our patient raised many provocations as multiple antibiotic regimens were used (as the first presumption of diagnosis was 
refractory pneumonia), blood transfusion was delayed due to the difficulty in preparing typed and cross-matched packed red blood cells and specific treatment of SLE with dexamethasone and medrol can significantly increase the risk of hypertension, gastritis and osteoporosis in a 78 diabetic and hypertensive patient.

Patients with older-onset SLE do not have a better outcome than the ones with a younger onset, even if they exhibit milder forms of disease than the last ones, they have even higher mortality rates (21).

\section{Conclusions}

Antibodies anti-dsDNA and antinuclear antibodies must be titred, and SLE must be taken into consideration even in elder male patients with hemoptysis, serositis and hemolytic anemia.

Therapeutic options in these patients are challenging because of the associated comorbidities and side effects.

\section{References}

1. Danchenko N, Satia JA, Anthony MS. Epidemiology of systemic lupus erythematosus: a comparison of worldwide disease burden. Lupus. 2006;15(5):308-18.

2. Achour A, Mankai A, Thabet Y, Sakly W, Braham F, Kechrid C, et al. Systemic lupus erythematosus in the elderly. Rheumatol Int. 2012;32(5):1225-9.

3. Almasi S, Ebrahimi N, Zamani K. A case report of old age systemic lupus erythematosus. Saman Publishing Co. 2015;3(4):74-6.

4. Yamaji K, Yasuda M, Yang KS, Kanai Y, Yamaji C, Kawanishi T, et al. A case of verylate-onset systemic lupus erythematosus. Mod Rheumatol. 2007;17(5):441-4.

5. Stefanidou S, Gerodimos C, Benos A, Galanopoulou V, Chatziyannis I, Kanakoudi $\mathrm{F}$, et al. Clinical expression and course in patients with late onset systemic lupus erythematosus. Hippokratia. 2013;17(2):1536.

6. Catoggio LJ, Soriano ER, Imamura PM, Wojdyla D, Jacobelli S, Massardo L, et al. Late-onset systemic lupus erythematosus in Latin Americans: a distinct subgroup? Lupus. 2015;24(8):788-95.

7. Ramos-Casals M, Brito-Zeron P, Lopez-Soto A, Font J. Systemic autoimmune diseases in elderly patients: atypical presentation and association with neoplasia. Autoimmun Rev. 2004;3(5):376-82.

8. Feng X, Zou Y, Pan W, Wang X, Wu M, Zhang $\mathrm{M}$, et al. Associations of clinical features and prognosis with age at disease onset in patients with systemic lupus erythematosus. Lupus. 2014;23(3):327-34.

9. Lockshin MD, Mary Kirkland Center for Lupus Research C. Biology of the sex and age distribution of systemic lupus erythematosus. Arthritis Rheum. 2007;57(4):608-11.

10. Hakim FT, Gress RE. Immunosenescence: deficits in adaptive immunity in the elderly. Tissue Antigens. 2007;70(3):179-89.

11. Ward MM, Polisson RP. A meta-analysis of the clinical manifestations of older-onset systemic lupus erythematosus. Arthritis Rheum. 1989;32(10):1226-32.

12. Pamuk ON, Balci MA, Donmez S, Tsokos GC. The incidence and prevalence of systemic lupus erythematosus in Thrace, 2003-2014: A 12-year epidemiological study. Lupus. 2016;25(1):102-9.

13. Rees F, Doherty M, Grainge MJ, Lanyon $\mathrm{P}$, Zhang W. The worldwide incidence and prevalence of systemic lupus erythematosus: a systematic review of epidemiological studies. Rheumatology (Oxford). 2017;56(11):1945-61.

14. Rovensky J, Tuchynova A. Systemic lupus erythematosus in the elderly. Autoimmun Rev. 2008;7(3):235-9.

15. Bogdan M, Tica I, Gheorghe DN, Silosi I, Solomon S, Martu I, et al. Effect of $0.2 \%$ Chlorhexidine's Use for Treatment of Localized Gingival Lesions in Patients with Type 2 Diabetes. Rev Chim(Bucharest). 2016;67(12):2651-3.

16. Haye Salinas MJ, Caeiro F, Saurit V, Alvarellos A, Wojdyla D, Scherbarth HR, et al. Pleuropulmonary involvement in patients with systemic lupus erythematosus from a Latin American inception cohort (GLADEL). Lupus. 2017;26(13):1368-77.

17. Dini AA, Wang P, Ye DQ. Serum Adiponectin 
Levels in Patients With Systemic Lupus Erythematosus: A Meta-analysis. J Clin Rheumatol. 2017;23(7):361-7.

18. Ionescu E, Tica I, Oprea C, Iliescu D, Petcu L, Iliescu M. Adiponectin Correlation With Bioclinical Benefits Of Using Natural Therapeutic Factors In Knee Osteoarthritis. Women. 2017;25(18.6):5.4.

19. Hashimoto H, Hirose S, Kano S, Tsunematsu T, Kabasawa K. [Studies on clinical subsets and severity of systemic lupus erythematosus based on a 1987 questionnaire conducted in Japan--clinical analysis of the outcome and treatments in clinical subsets]. Ryumachi. 1992;32(1):27-38.

20. Choi J, Park D, Kang J, Yim Y, Lee K, Lee J, et al. Comparison of clinical and serological differences among juvenile-, adult-, and late-onset systemic lupus erythematosus in Korean patients. Lupus. 2015;24(12):13429.

21. Tomic-Lucic A, Petrovic R, RadakPerovic M, Milovanovic D, Milovanovic $\mathrm{J}$, Zivanovic S, et al. Late-onset systemic lupus erythematosus: clinical features, course, and prognosis. Clin Rheumatol. 2013;32(7):1053-8. 